\title{
ON PRIME AND SEMIPRIME NEAR-RINGS WITH DERIVATIONS
}

\author{
NURCAN ARGAÇ \\ Ege University \\ Science Faculty \\ Department of Mathematics \\ 35100 Bornova, Izmir, TURKEY \\ e-mailEfemat01@vm3090 ege edu.tr
}

(Received July 10, 1995 and in revised form April 16, 1996)

\begin{abstract}
Let $N$ be a semiprime right near-ring, $A$ a subset of $N$ such that $0 \in A$ and $A N \subseteq A$, and $d$ a derivation of $N$ The purpose of this paper is to prove that if $d$ acts as a homomorphism on $A$ or as an anti-homomorphism on $A$, then $d(A)=\{0\}$
\end{abstract}

KEY WORDS AND PHRASES: Prime near-ring, semiprime near ring, ideal, derivation, homomorphism, anti-homomorphism

1991 AMS SUBJECT CLASSIFICATION CODES: 16 Y30.

\section{INTRODUCTION}

Throughout this paper $N$ will be a right near-ring A derivation on $N$ is defined to be an additive endomorphism satisfying the "product rule" $d(x y)=x d(y)+d(x) y$ for all $x, y \in N$ According to Bell and Mason [1], a near-ring $N$ is said to be prime if $x N y=\{0\}$ for $x, y \in N$ implies $x=0$ or $y=0$, and semiprime if $x N x=\{0\}$ for $x \in N$ implies $x=0$ Let $S$ be a nonempty subset of $N$ and $d$ be a derivation of $N$ If $d(x y)=d(x) d(y)$ or $d(x y)=d(y) d(x)$ for all $x, y \in S$, then $d$ is said to act as a homomorphism or anti-homomorphism on $S$, respectively. As for terminologies used here without mention, we refer to Pilz [2]

Bell and Kappe [3] proved that if $d$ is a derivation of a semiprime ring $R$ which is either an endomorphism or anti-endomorphism, then $d=0$ They also showed that if $d$ is a derivation of a prime ring $R$ which acts as a homomorphism or an anti-homomorphism on $I$, where $I$ is a nonzero right ideal, then $d=0$ on $R$

\section{THE RESULTS}

It is our aim in this paper to prove that the above conclusions hold for near-rings as follows

THEOREM Let $N$ be a semiprime right near-ring, and $d$ a derivation on $N$ Let $A$ be a subset of $N$ such that $0 \in A$ and $A N \subseteq A$ If $d$ acts as a homomorphism on $A$ or as an anti-homomorphism on $A$, then $d(A)=\{0\}$.

In order to give the proof of the above theorem we need the following lemmas

LEMMA 1. If $N$ is a right near-ring and $d$ a derivation of $N$, then

$$
c(y d(x)+d(y) x)=c y d(x)+c d(y) x \text { for all } x, y, c \in N .
$$

A proof can be given by using a similar approach as in the proof of [1, Lemma 1] 
LEMMA 2. Let $N$ be a right near-ring, $d$ a derivation of $N$, and $A$ a multiplicative subsemigroup of $N$ which contains 0 If $d$ acts as an anti-homomorphism on $A$, then $a 0=0$ for all $a \in A$

PROOF. Since $0 a=0$ for all $a \in A$ and $d$ acts as an anti-homomorphism on $A$ then we have $d(a) 0=0$ for all $a \in A$ Taking $a 0$ instead of $a$, one can obtain $a 0+d(a) 0=0$ for all $a \in A$ Thus we get $a 0=0$ for all $a \in A$

LEMMA 3. Let $N$ be a right near-ring, and $A$ a multiplicative subsemigroup of $N$

(a) If $d$ acts as a homomorphism on $A$, then

$$
d(y) x d(y)=y x d(y)=d(y) x y \text { for all } x, y \in A .
$$

(b) If $d$ acts as an anti-homomorphism on $A$, then

$$
d(y) x d(y)=d(y) y x=x y d(y) \text { for all } x, y \in A .
$$

PROOF. (a) Let $d$ act as a homomorphism on $A$ Then

$$
d(x y)=x d(y)+d(x) y=d(x) d(y) \text { for all } x, y \in A .
$$

Taking $y x$ instead of $x$ in (2.3) we get

$$
y x d(y)+d(y x) y=d(y x) d(y)=d(y) d(x y) \text { for all } x, y \in A .
$$

By Lemma $1, d(y) d(x y)=d(y) x d(y)+d(y) d(x) y=d(y) x d(y)+d(y x) y$ Using this relation in (24), we obtain $y x d(y)=d(y) x d(y)$ for all $x, y \in A$ Similarly, taking $y x$ instead of $y$ in (23) one can prove the relation $d(y) x d(y)=d(y) x y$ for all $x, y \in A$.

(b) Since $d$ acts as an anti-homomorphism on $A$, we have

$$
d(x y)=x d(y)+d(x) y=d(y) d(x) \text { for all } x, y \in A .
$$

Substituting $x y$ for $y$ in (25) leads to

$$
\begin{aligned}
x d(x y)+d(x) x y & =d(x y) d(x) \\
& =x d(y) d(x)+d(x) y d(x) \\
& =x d(x y)+d(x) y d(x) \quad \text { for all } \quad x, y \in A .
\end{aligned}
$$

From this relation we arrive at $d(x) x y=d(x) y d(x)=0$ for all $x, y \in A$ Similarly taking $x y$ instead of $x$ in (25), one can prove the relation $d(y) x d(y)=x y d(y)$ for all $x, y \in A$.

PROOF OF THEOREM. (a) First suppose that $d$ acts as a homomorphism on $A$. By Lemma 3 (a), we have

$$
d(y) x d(y)=d(y) x y \text { for all } x, y \in A .
$$

Right-multiplying (2.6) by $d(z)$, where $z \in A$, and using the hypothesis that $d$ acts as a homomorphism on $A$ together with Lemma 1, we obtain $d(y) x d(y) z=0$ for all $x, y, z \in A$ Taking $x r$ instead of $x$, where $r \in N$, we have $d(y) x r d(y) z=0$ for all $x, y, z \in A$ and $r \in N$. Hence $d(y) x N d(y) x=\{0\}$ for all $x, y \in A$; and by semiprimeness

$$
d(y) x=0 \text { for all } x, y \in A .
$$

Substituting $y r$ for $y$ in (27), where $r \in N$, leads to

$$
y d(r) x+d(y) r x=0 \text { for all } x, y \in A, r \in N .
$$

Left-multiplying (2.8) by $d(z)$, where $z \in A$, we have that $d(z) y d(r) x+d(z) d(y) r x=0$ According to (2 7) this relation reduces to $d(z y) r x=0$. Hence we get $z d(y) r x=0$ for all $x, y, z \in A$ and $r \in N$ By semiprimeness, we get

$$
z d(y)=0=z r d(y) \text { for all } y, z \in A \text { and } r \in N .
$$


Combining (2 7) and (2.9) shows that $d(y z)=0$ for all $y, z \in A$ In particular, $d(x r x)=0$ for all $x \in A, r \in N$, and since $d$ acts as a homomorphism on $A$,

$$
d(x r) d(x)=0=x d(r) d(x)+d(x) r d(x) \text { for all } x \in A, r \in N .
$$

In view of (2.9), this gives $d(x) N d(x)=\{0\}$ and hence $d(x)=0$ for all $x \in A$

(b) Now assume that $d$ acts as an anti-homomorphism on $A$ Note that $a 0=0$ for all $a \in A$ by Lemma 2. According to Lemma 3 (b),

$$
\begin{aligned}
& d(y) x d(y)=x y d(y) \text { for all } \quad x, y \in A, \\
& d(y) x d(y)=d(y) y x \text { for all } \quad x, y \in A .
\end{aligned}
$$

Replacing $x$ by $x d(y)$ in (2.10) and using Lemma 1 we get

$$
d(y) x y d(y)+d(y) x d(y) y=x d(y) y d(y) \text { for all } x, y \in A .
$$

Substituting $x y$ for $x$ in (210), we have

$$
d(y) x y d(y)=x y^{2} d(y) \text { for all } x, y \in A .
$$

Right-multiplying $(210)$ by $y$ we arrive at

$$
d(y) x d(y) y=x y d(y) y \text { for all } x, y \in A .
$$

Replacing $x$ by $y$ in (2.10), we have $d(y) y d(y)=y^{2} d(y)$, and left-multiplying this relation by $x$, we obtain

$$
x d(y) y d(y)=x y^{2} d(y) \text { for all } x, y \in A .
$$

Using (2 13), (2 14), and (2.15) in (2.12) one obtains $x y d(y) y=0$ for all $x, y \in A$, hence $y r y d(y) y=0$ and $y d(y) y r y d(y) y=y d(y) 0=0$ for all $y \in A, r \in N$, and by semiprimeness

$$
y d(y) y=0 \text { for all } y \in A .
$$

According to (2.14) we get $d(y) x d(y) y=0$ for all $x, y \in A$ Using this relation in (2 11), we arrive at

$$
d(y) y x y=0 \text { for all } x, y \in A \text {. }
$$

Replacing $x$ by $x d(y)$ in (2.16), we have $d(y) y x d(y) y=0=d(y) y x r d(y) y x$ for all $x, y \in A, r \in N$, hence

$$
d(y) y x=0 \text { for all } x, y \in A .
$$

Using (2 17) in (2 11), one obtains $d(y) x d(y)=0=d(y) x r d(y) x$ for all $x, y \in A, r \in N$, hence

$$
d(y) x=0 \text { for all } x, y \in A \text {. }
$$

Therefore,

$$
\begin{array}{lll}
x d(z) d(y n) x=0 & \text { for all } & x, y, z \in A, n \in N, \\
x d(z)(y d(n)+d(y) n) x=0 & \text { for all } & x, y, z \in A, n \in N, \quad \text { and } \\
x d(z) y d(n) x+x d(z) d(y) n x=0 & \text { for all } & x, y, z \in A, n \in N .
\end{array}
$$

In view of (2 18), this gives $x d(z) d(y) n x=0=x d(z) d(y) n x d(z) d(y)$, hence $x d(z) d(y)=0$ for all $x, y, z \in A$ Since $d$ acts as an anti-homomorphism on $A$, we have $x d(y z)=0$ for all $x, y, z \in A$, so that $x y d(z)+x d(y) z=0$ for all $x, y, z \in A \quad$ By (2.18) we now get $x y d(z)=0=x d(z) r y d(z)$ for all $x, y, z \in A$ and $r \in N$; and taking $x$ instead of $y$ we get $x d(z)=0$ for all $x, z \in A$ Recalling (2.18), we now have $d(x y)=0$ for all $x, y \in A$, so $d(x x r)=0$ for all $x \in A$ and $r \in N$. Thus $d(x r) d(x)=0$, and we finish the proof as in case (a).

We now state some consequences of the theorem 
COROLLARY 1. Let $N$ be a semiprime right near-ring, and $d$ a derivation of $N$ If $d$ acts as a homomorphism on $N$ or as an anti-homomorphism on $N$, then $d=0$

COROLLARY 2. Let $N$ be a prime right near-ring, and $d$ a derivation of $N$ Let $A$ be a nonzero subset of $N$ such that $0 \in A$ and $A N \subseteq A$. If $d$ acts as a homomorphism on $A$ or as an antihomomorphism on $A$ then, $d=0$

PROOF. By the theorem, we have $d(a)=0$ for all $a \in A$ Then $d(a x)=a d(x)+d(a) x=a d(x)=$ $0=\operatorname{ayd}(x)$ for all $a \in A, x, y \in N$, and by primeness we get $a=0$ or $d(x)=0$ for all $a \in A, x \in N$ Since $A$ is nonzero, we have $d(x)=0$ for all $x \in N$

ACKNOWLEDGMENT. The author would like to thank the referee and Prof $\mathrm{H}$ E Bell for valuable suggestions

\section{REFERENCES}

[1] BELL, H.E and MASON, G., On derivations in near-rings, Near-rings and Near-fields, NorthHolland Mathematics Studies 137 (1987), 31-35

[2] PILZ, G, Near-Rings (2nd edition), North-Holland, Amsterdam-New York-Oxford, 1983

[3] BELL, H E. and KAPPE, L.C., Rings in which derivations satisfy certain algebraic conditions, Acta Math. Hungar. 53 (1989), no. 3-4, 339-346 


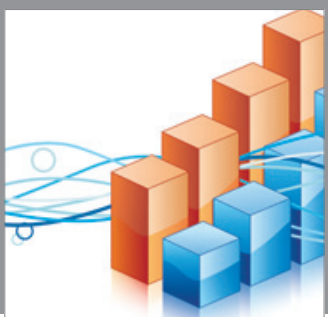

Advances in

Operations Research

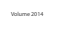

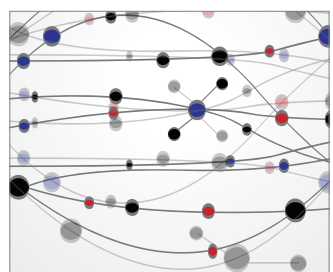

\section{The Scientific} World Journal
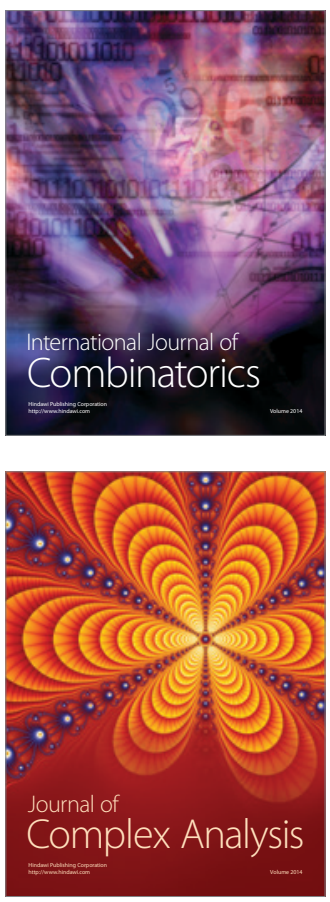

International Journal of

Mathematics and

Mathematical

Sciences
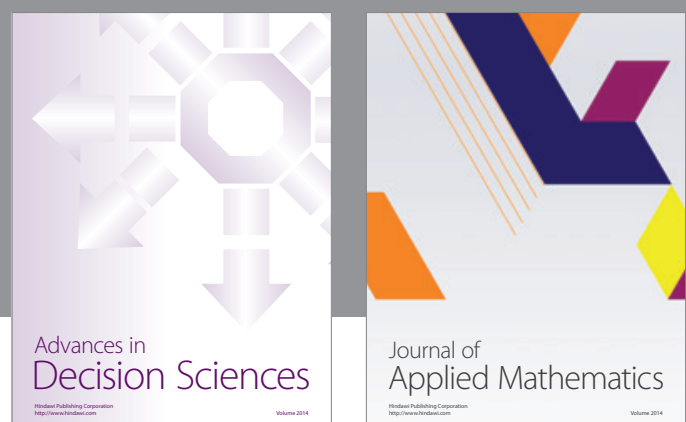

Journal of

Applied Mathematics
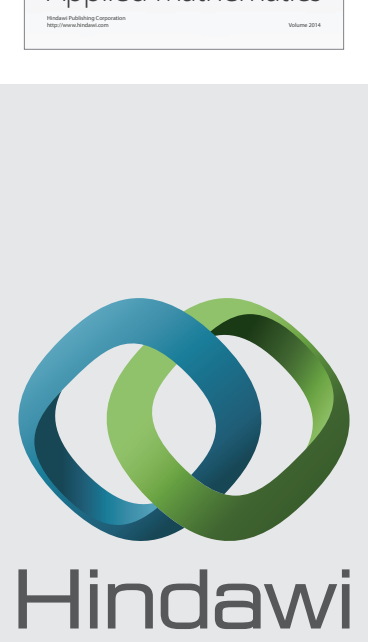

Submit your manuscripts at http://www.hindawi.com
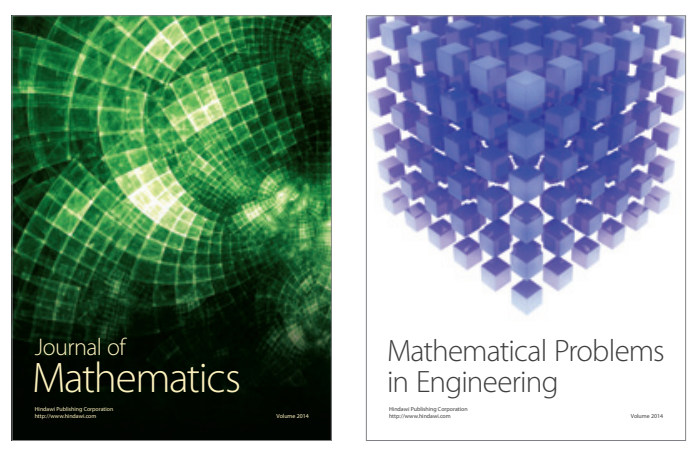

Mathematical Problems in Engineering
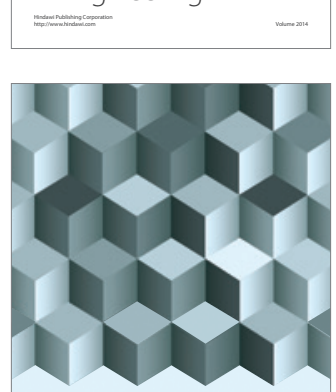

Journal of

Function Spaces
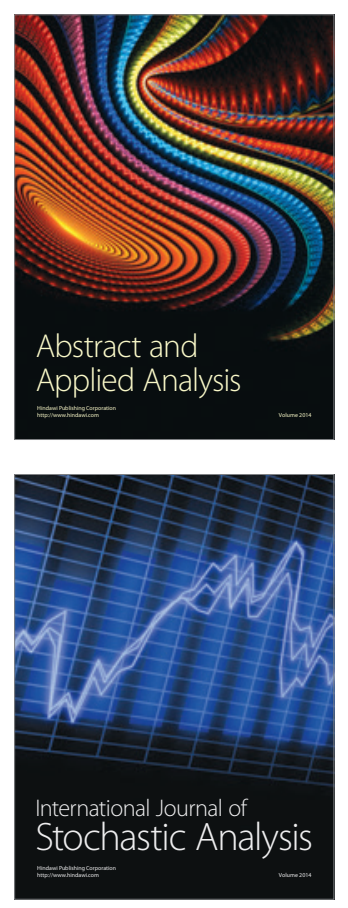

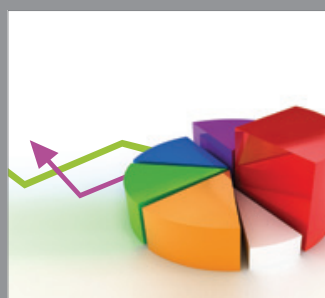

ournal of

Probability and Statistics

Promensencen
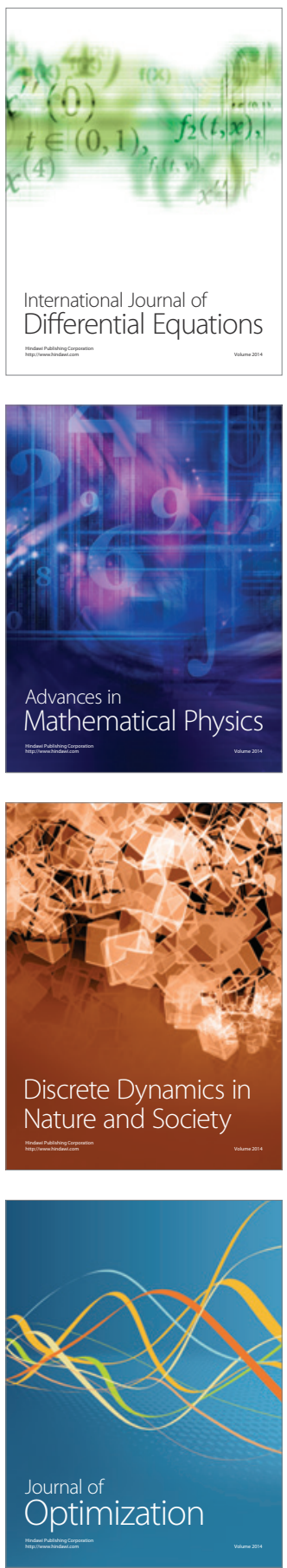